\title{
Research and realization of automatic test system for motor controller unit
}

\author{
Lingxiao Zhao ${ }^{1,2}$, Xiangpeng Zeng ${ }^{1,2,}$, and Huansheng $\mathrm{Ma}^{1,2}$ \\ ${ }^{1}$ CATARC Automotive Test Center (Tianjin) Co., Ltd, Tianjin 300300, China \\ ${ }^{2}$ China Automotive Technology \& Research Center Co., Ltd, Tianjin 300300, China
}

\begin{abstract}
The development of electric vehicles has gradually become the mainstream trend of the automobile industry, and the motor controller unit is one of the key parts of the electric vehicle. The reliability and safety of the electric vehicle motor controller unit have attracted extensive attention in the industry in recent years. In order to meet the automotive industry on the motor controller unit reliability test verification. This paper studies and designs an automatic test system of the motor controller unit. And introduces the hardware and software control strategy test system in detail. The test results prove the feasibility and convenience of the test system.
\end{abstract}

Keywords: Electric vehicle; Motor controller unit; Automatic test system.

\section{Preface}

In recent years, electric vehicles have developed rapidly and become one of the alternative ways for people to travel. Therefore, their safety and reliability have attracted extensive attention[1].As one of the core power components of an electric vehicle, the motor controller unit can drive the electric vehicle motor and control the voltage and current of the power motor[2].With electric vehicle development, the requirements for the power drive system of motor controller unit to improve [3], and the reliability is one of the important indicators to measure motor controller unit, in its development should be fully confirmed the reliability of the product design, through a longer period of more stringent conditions of test evaluation, to ensure the reliability of the motor controller unit, thereby promoting the sustained and healthy development of the electric vehicle power system.

At present in view of the motor controller unit test evaluation mainly from the functional resistance to salt fog environment aspects such as reliability, durability and mechanical properties in [4],the test project has a long time period sampling data consistency test equipment and controller under test samples more demanding characteristics $[5,6]$ at present ,the domestic professional testing institutions, generally the motor controller unit and peripheral test equipment, such as environmental box salt fog cooling system power control separately, this test method is more shortage, such as not synchronous equipment and sample status Therefore,to research and design the automatic test system of motor controller unit is a great significance.

\footnotetext{
*Corresponding author: zengxiangpeng@catarc.ac.cn
} 
Based on CANoe(CAN Open Environment) software, this paper uses C\# programming to control test equipment and CAN bus to communicate with motor controller unit in real time, The analog signals such as temperature and voltage are collected and fed back through I /O interface, In order to ensure the consistency and real-time of the data between the test sample and the test equipment, a set of automatic test system is designed, and the feasibility of the design scheme is verified by the test, which can realize the automatic test of the reliability of the motor controller unit.

\section{System design and principle}

The automatic test system designed in this paper mainly includes hardware system composition and software system architecture. Based on CAE language, the motor control system is designed to simulate the working condition of the whole system.

\subsection{Hardware system}

The hardware system mainly includes high-voltage DC power supply, low-voltage DC power supply, inductive load, cooling system, environment box, I/O interface, etc. Among them, the high-voltage DC power supply is used to simulate the power battery of electric vehicle to provide DC power supply for motor controller unit, with voltage level of $800 \mathrm{~V}$ and power level of $30 \mathrm{~kW}$, which can provide smooth and stable DC output to charge the bus capacitor of motor controller unit; the low-voltage DC power supply simulates the lowvoltage battery, with voltage level of $30 \mathrm{~V}$ and power of $100 \mathrm{~W}$, which provides low-voltage control circuit power supply for motor controller unit To meet the working environment of motor controller unit control board; inductive load simulation drive motor coil, with 150uh,300uh and other specifications, support current frequency of $0-1000 \mathrm{hz}$, continuous peak current of $600 \mathrm{~A}$, as the load of the controller, to form a current output circuit; cooling system simulation of the whole vehicle cooling cycle system, temperature range of $-40{ }^{\circ} \mathrm{C}$ $90{ }^{\circ} \mathrm{C}$, temperature change rate of $10{ }^{\circ} \mathrm{C} / \mathrm{min}$, to provide reference for motor control Circulating cooling, and meet the rapid change of cooling temperature under more stringent conditions, to raise or lower the temperature for the sample of motor controller unit; The environment box simulates the real vehicle environment, the temperature range is $-50{ }^{\circ} \mathrm{C}-120{ }^{\circ} \mathrm{C}$, the temperature change rate is $15{ }^{\circ} \mathrm{C} / \mathrm{min}$, and the humidity range is $20 \%$ $100 \%$. It provides the temperature and humidity conditions for the motor controller unit, and can simulate the extremely harsh environmental conditions. The upper computer system can simulate the electric vehicle controller and other key control nodes of the whole vehicle, and send the working mode, output current, output current, etc. to the motor controller unit through CAN bus At the same time, it can receive analog data such as temperature, current and voltage feedback from the sample through I /O interface.

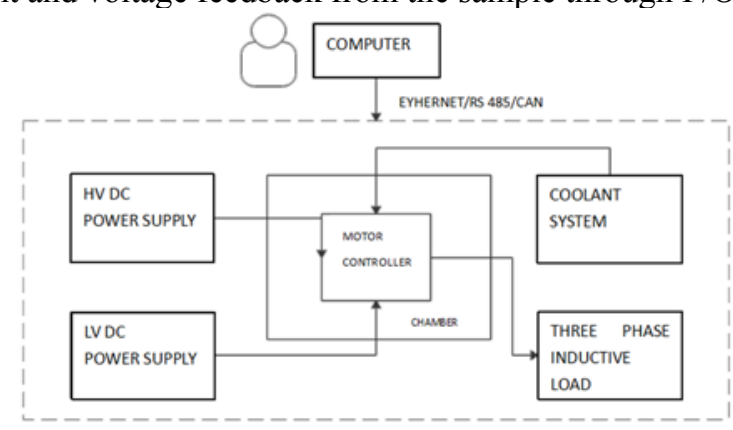

Fig. 1. Hardware structure of automatic control system. 


\subsection{Software system}

The software system is developed based on canoe, which is a bus analysis software designed and developed by vector company of Germany. It supports can bus, canfd, Lin, Ethernet and other vehicle communication modes, and is widely used in vehicle bus network simulation analysis, test and development [7,8]. Software system architecture based on canoe and C \# programming language development [9].

Firstly, the simulation test network is established in canoe, and each hardware node is added to simulate the test network environment.

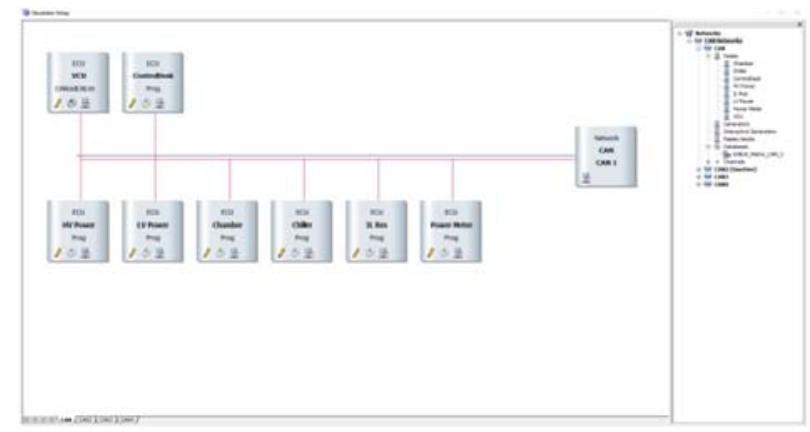

Fig. 2. Simulation environment of ATS.

Then, based on the communication protocol of each hardware device, the system variables for the device are established, and the interaction program between each hardware device and canoe is written with $\mathrm{C}$ \#. The system variables are established, and canoe sends instructions to interact with the device. Due to the number and variety of integrated devices, and the high real-time requirements for each action response, a separate thread is created for each device, and the new thread processes the sending and receiving of programcontrolled instructions to avoid thread blocking.

The program execution logic is mainly divided into two aspects, one is the main thread code execution logic in canoe, the other is the new thread code execution logic.

As shown in Figure 3, the main thread code execution logic of canoe is mainly related to the event driven by the change of system variable value. If the device has been successfully connected, when a system variable event is generated, the corresponding instruction will be put into the execution queue.

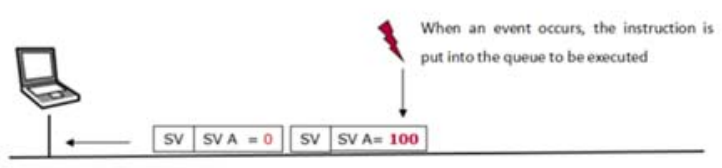

Fig. 3. Main thread execution logic.

As shown in Figure 4, it is the execution logic of the device thread code. After the connection is established, the instructions in the queue are processed in a cycle from start to stop. The execution cycle can be adjusted according to the dynamic response characteristics of the test requirements to adapt the test requirements of different action frequencies. 


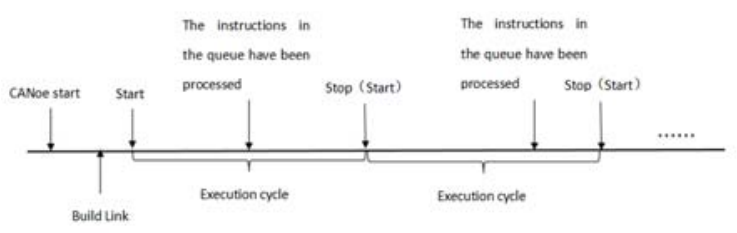

Fig. 4. Multithreading execution logic.

Finally, based on the pannel designer tool in canoe, the key parameters and control switches used in the test are summarized, and the hardware device control components and control interface are constructed. The hardware devices are controlled in the upper computer interface, and the protection and alarm components are set. When there is a process that does not follow the test case in the test process, it can alarm and respond to the protection action in time.

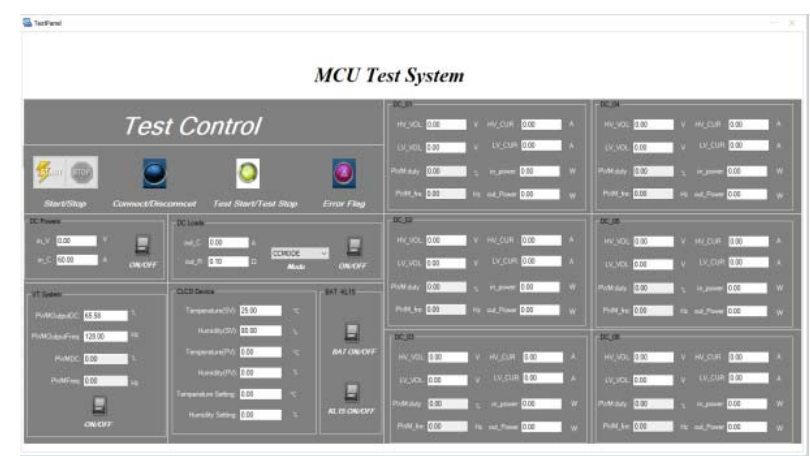

Fig. 5. Test and monitoring interface.

\section{Develop test cases}

At present, the reliability test of motor controller unit is mainly based on ISO 16750 and GB / T 28046,vw80300,GMW3172 and the enterprise regulations of domestic and foreign main engine manufacturers can be divided into temperature cycle reliability, high temperature durability reliability, cycle damp heat reliability and other test conditions according to different test and evaluation emphases of products; in addition, some tests need to be conducted according to the battery voltage, ambient temperature, motor speed, driving current and other main parameters of real vehicle conditions In order to find the design defects in the motor controller unit development stage, locate the product problems and improve the reliability of the product, the real vehicle working conditions are simulated in the laboratory and the conditions are reproduced.

According to the test control strategy, the reliability test of the motor controller unit can be divided into Open-loop test and closed-loop test. The Open-loop test requires that the environment temperature, humidity, output current and other variables of the motor controller unit change with time, and there is no coupling relationship between them or the coupling relationship is not strong; the closed-loop test requires that the internal temperature of the motor controller unit be taken as the target quantity, and the internal temperature can be balanced by controlling the output current or the environment temperature. 


\subsection{Open loop control test}

In the traditional national standards and industry standards for the reliability test requirements of motor controller unit, the change of test conditions is only related to the working mode and output state of motor controller unit with time. As shown in the figure below, one cycle lasts for 8 hours. In the final stage of low temperature, the product enters the primary working mode after reaching the lowest temperature. In the heating stage, the product enters the primary working mode from $20{ }^{\circ} \mathrm{C}$ to the end of high temperature. There is no coupling relationship between various parameters.

For this kind of reliability test conditions, open-loop control method is adopted to control the test variables changing with time in real time. Vtest studio and automation programming module are used to realize the development of Open-loop test cases.

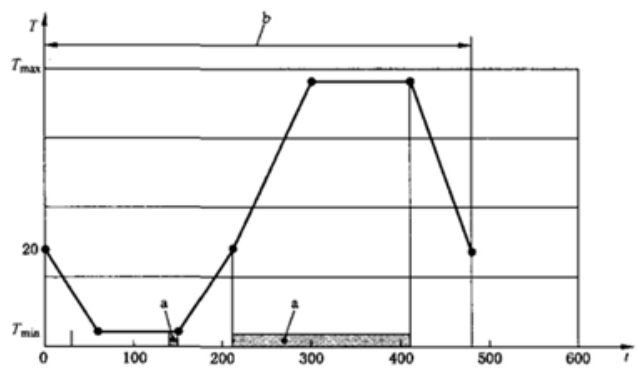

Fig. 6. Temperature cycle test conditions.

\subsubsection{Based on vtest studio}

Vtest studio is a test software developed by vector for test sequence management, which is used to develop test cases and form a complete test system with canoe. Among them, vtest studio can directly associate the system variables in canoe, and directly set the time-varying curve of the relevant variables, which is transmitted to the equipment and motor controller unit through the general network, so as to realize the automatic operation of test cases.

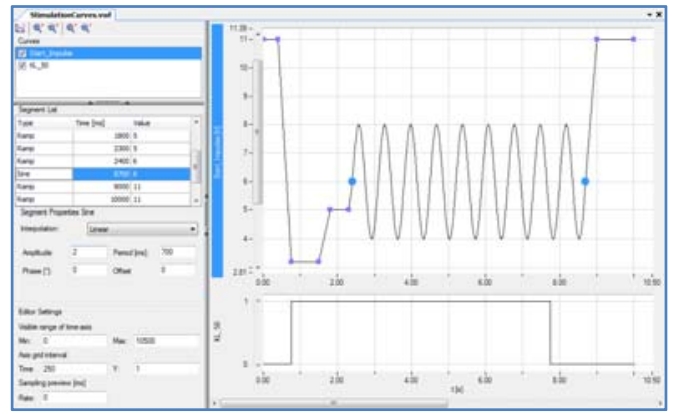

Fig. 7. Vtest studio test curve editing.

\subsubsection{Based on automatuon module}

Using automation in canoe software, the system variables can be set linearly according to the test cases, and the test cases can be run automatically. The test flow chart is shown in the figure below. 


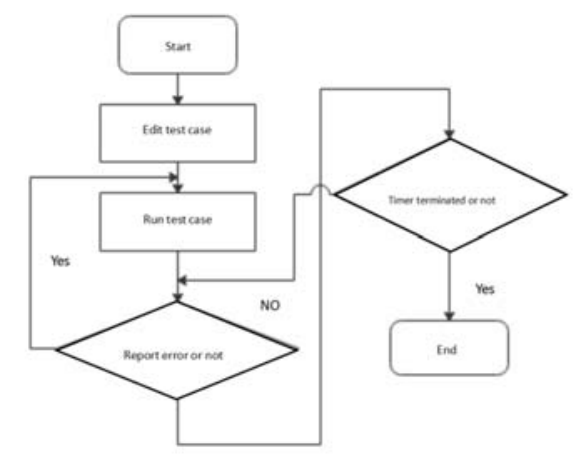

Fig. 8. Flow chart of automation test based on Automation.

\subsection{Closed loop control test}

In addition to the requirements of traditional national standards and industry standards, the reliability of the motor controller unit in the industry is constantly improving. Combined with the real vehicle conditions, the motor controller unit can work in the temperature balance condition for a long time. For this kind of reliability test, the closed-loop control strategy is adopted, and the temperature of PCB or IGBT in the motor controller unit is taken as the controlled variable to ensure that the motor controller unit can work at the temperature equilibrium point.

In this paper, the output current and the temperature of the cooling system are controlled to keep the temperature constant when the temperature changes. The above variables are controlled by programming in CAPL language in canoe. The program flow chart is as follows.

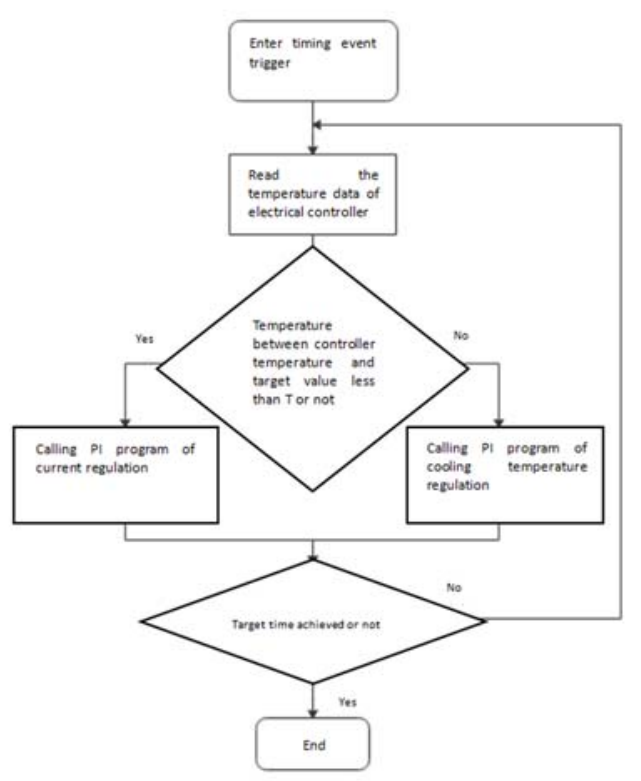

Fig. 9. Closed loop control flow chart. 
Because temperature is a variable with large inertia, double closed-loop control of cooling system temperature and controller output current is adopted. A timer trigger event is set to adjust the temperature periodically. When the difference between the actual temperature and the target temperature is small, PI is used to adjust the output current of the motor controller unit, so as to adjust the heating power of the controller to achieve the purpose of slowly adjusting the temperature. When the difference between the actual temperature and the target temperature is large, or the purpose of temperature adjustment can not be achieved by adjusting the output current, PI is used to adjust the temperature. The temperature of the cooling system can quickly transfer heat with the controller, so as to achieve the purpose of rapid regulation. The control block diagram is shown below.

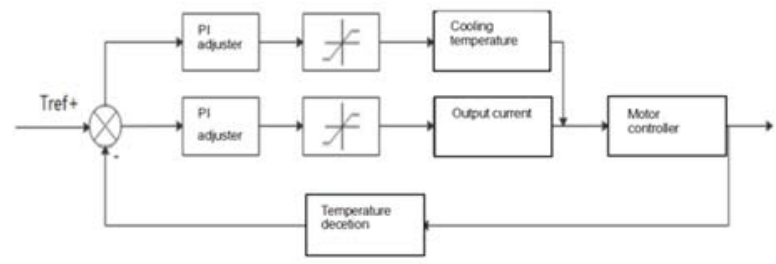

Fig. 10. Temperature control block diagram of controller.

\section{Test verification}

In order to verify the feasibility of the automatic test system designed in this paper, we developed and built the automatic test system as shown in Figure 11, and the motor controller unit is tested and verified according to different test methods. The feasibility of the test system under different test conditions is verified by collecting the data of environment temperature, cooling system temperature, motor controller unit high voltage, output current and so on it's not easy.

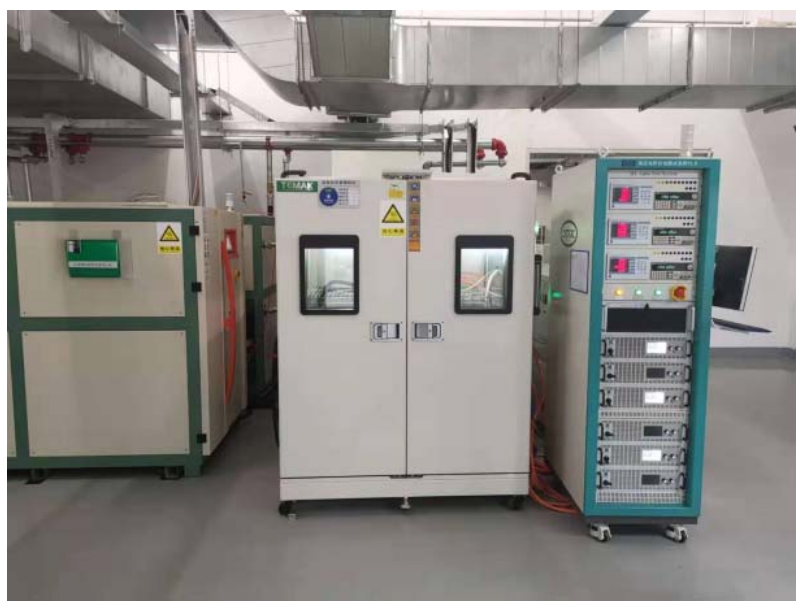

Fig. 11. Automation test system.

Figure 12 is the temperature cycle reliability test curve collected by using open-loop control method and based on vtest studio programming. Among them, the system variables are sent to change the high-voltage voltage, ambient temperature, cooling temperature, etc., 
and messages are sent through CAN bus to adjust the output current of motor controller unit, so that the parameters change with time according to the set curve.

It can be seen that the ambient temperature can change according to the set rule, and at the required temperature point and time, the motor controller unit can output the corresponding current, which meets the test conditions.

Figure 13 is a test case based on automaiton. Under different high-voltage input voltages, the output current of the motor controller unit is continuously switched. The test data show that the test sample can operate automatically according to the set instructions and switch the state as required.

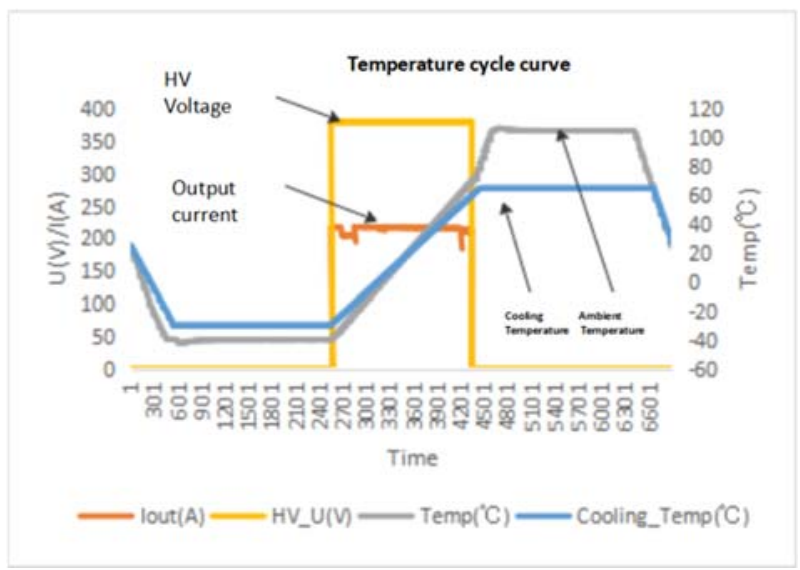

Fig. 12. Temperature cycle test curve.

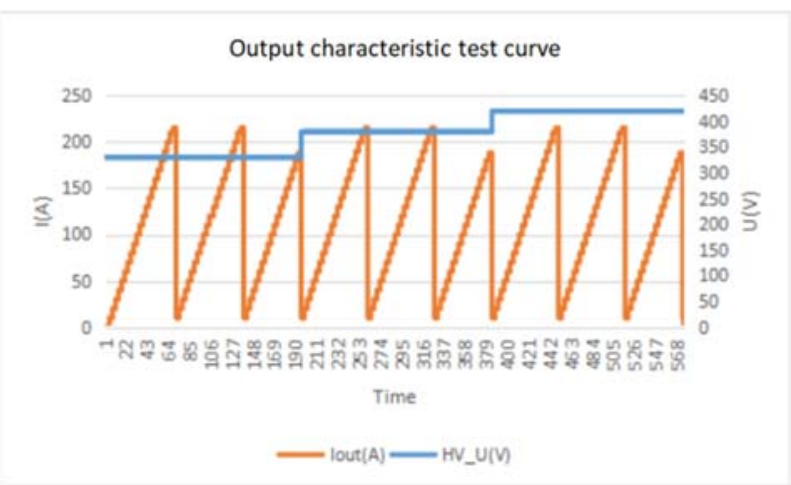

Fig. 13. Output characteristic test curve.

Figure 14 shows the high temperature reliability test of the motor controller unit by using the closed-loop control method. In order to ensure that the motor controller unit can work in the thermal stable state for a long time, the output current is adjusted in real time, so as to adjust the output power, and the reliability of the controller under the steady-state condition is investigated. 


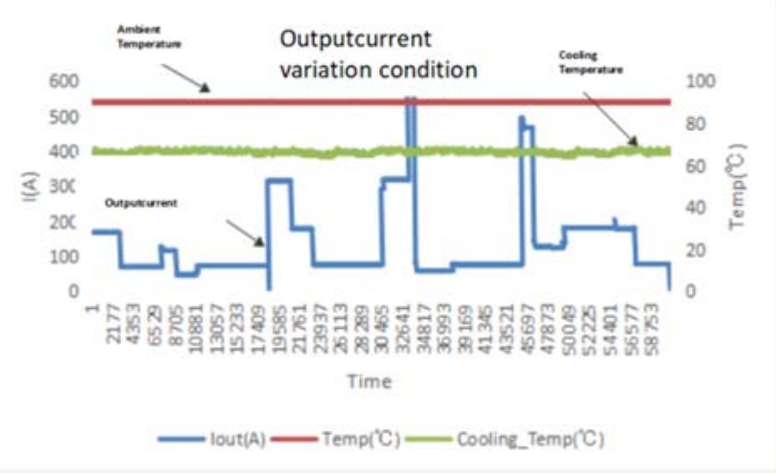

Fig. 14. Working condition curve of circulating current.

\section{Conclusion}

The automatic test system of motor controller unit designed in this paper is based on the needs of the industry, based on canoe environment, using $\mathrm{C} \#$ to write the underlying software architecture, through Ethernet, RS485, can bus and other communication methods, it can control the motor controller unit and hardware equipment system to be tested in real time, and collect real-time test data. Based on the open-loop control strategy, it can achieve national standards and industry standards In order to meet the industry's more stringent reliability testing requirements for motor controller unit, the system can simulate the real vehicle working conditions and conduct closed-loop control of hardware parameters and motor controller unit working mode.

\section{References}

1. Shi Zhili, Hou Feiyue. Discussion on the driving motor of new energy vehicles [J]. Automotive practical technology,2019 (24): 19-21

2. Nie Guanghui. Control principle of electric vehicle motor controller unit [J]. Modern industrial economy and informatization,2019,9 (11): 56-57

3. Wang Dan, Xu Dan, Cao Ruigang, et al. Overview on key techniques of electric vehicle[J]. Engineering Science,2013,(1):68-72(in Chinese).,2013,(1):68-72.

4. Cao Dongdong, $\mathrm{Hu}$ Jian, $\mathrm{Xu}$ Xiao. Research on standard system of electric drive system for electric vehicles [J]. China automotive,2019 (04): 50-54

5. Liu Jun, Zhong Yulin, Zhang Jin, Wen Xuhui. Research and development of high power density motor controller unit for electric vehicles [J]. Power electronics technology,2011,45 (12): 14-16

6. Zhang Ling, Dong Ruixin, Zhang Hongtao. Design of cooling system for electric vehicles [J]. Automotive electronics,2017 (02): 1-4

7. Qin Huaqiang, Su Kai, Zhou Zhiliang. Design of CAN bus gateway test model based on CANoe [J]. Automotive electronics,2019 (05): 48-51

8. Shen Hongyu, song Jingang, song Jinming. Construction of BCM test system based on canoe and VT system [J]. Automotive practical technology,2016 (10): 158-160 + 175

9. Wang Yonghui. Application of canoe in vehicle system development and test $[\mathrm{J}]$. Automotive practical technology,2019 (16): 85-87 